\title{
Automated mapping of the crystallographic sample orientation from diffraction patterns in momentum-resolved STEM
}

\author{
Mauricio Cattaneo ${ }^{1}$, Knut Müller-Caspary ${ }^{1}$, Juri Barthel ${ }^{1}$, Katherine MacArthur ${ }^{2}$ and Marta Lipinska-Chwalek ${ }^{1}$ \\ ${ }^{1}$ Ernst Ruska Centre for Microscopy and Spectroscopy with Electrons and the Peter Grünberg Institute \\ Forschungszentrum Jülich, 52425 Jülich, Germany, United States, ${ }^{2}$ Ernst Ruska Centre for Microscopy and \\ Spectroscopy with Electrons and the Peter Grünberg Institute Forschungszentrum Jülich, 52425 Jülich, Germany, \\ Germany
}

In scanning transmission electron microscopy (STEM), sample tilt with respect to the incident electron beam is a parameter that we want to control precisely to obtain optimum imaging conditions. However, residual tilts cannot always be eliminated because of the limited precision of the tilt stage and because they are partly due to the sample geometry at interfaces or bent specimens. In this study, we present an automated method for measuring local crystal orientation from convergent beam electron diffraction patterns. Using ultrafast cameras that record a diffraction pattern at each scan position (4D STEM), mapping of crystal orientation over a field of view becomes possible. This improves the quality of available knowledge about the sample compared to traditional STEM where sample tilt was measured at only one position, if at all. The availability of tilt mapping has the potential to improve strain measurements [1], spectroscopy at interfaces [2], and quantitative studies of bent samples that require local sample tilt as input to simulations.

Central to our method is the detection of Kikuchi band crossings in diffraction patterns to accurately locate the Laue circle. The distance of Kikuchi band crossings from the central beam of the incident probe is used as a measure of out-of-plane inclination, while a mutual change of azimuthal orientation of all bands around a crossing provides information about in-plane rotation. Band positions are determined from peaks found in azimuthal scans of an annular region at large scattering angles, as shown in Fig. 1. The position of the main band intersection is estimated and iteratively refined by adjusting the annular area. This procedure has been implemented in a fast computer program and can be automatically applied to a series of patterns as recorded in a 4D STEM experiment.

Achievable accuracy and precision were investigated by simulations. For realistic parameters related to the detector and electron dose used, the method was found to be accurate within $0.1 \mathrm{mrad}$ for the out-ofplane orientation and 5mrad for the in-plane orientation for a typical averaging of diffraction patterns over a unit cell. The simulations also show that the spatial resolution of this approach is limited by the lateral extent of probe propagation in the crystal. In case of e.g. [110] Fe, the electron probe extends laterally over 5 unit cells at 40nm sample thickness.

The method is applied to 4D-STEM data acquired from a twin boundary in steel using an Electron Microscope Pixel Array Detector (EMPAD) in a probe-corrected FEI Titan microscope operated at an accelerating voltage of $200 \mathrm{kV}$. The analysis provides a map of position-dependent local crystal orientation with unit cell sampling and reproduces the expected in-plane rotation of $70.53^{\circ}$ between the twins, as shown in Fig. 2. Due to the speed of the implemented measurement procedure, such analysis is 
possible within minutes and provides immediate insight into the presence of sample bending, strain fields, and stacking faults that were not possible prior to the advent of momentum-resolved STEM.

(a)

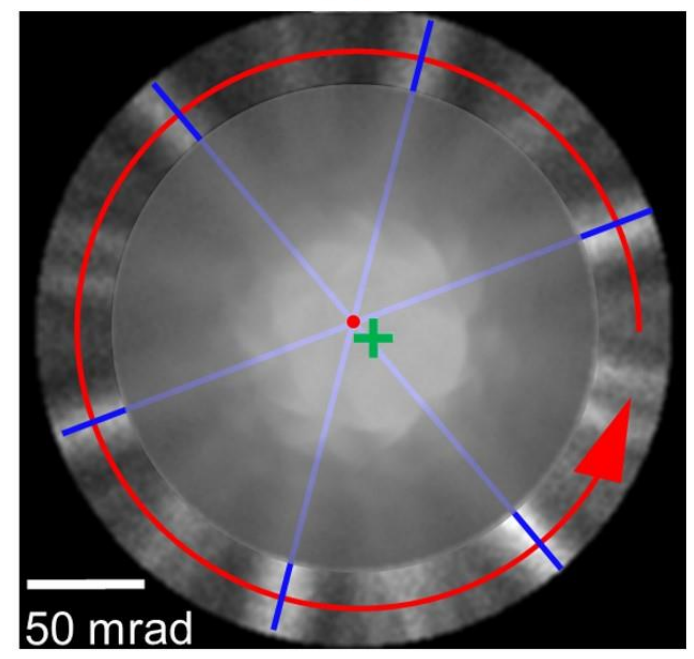

(b)

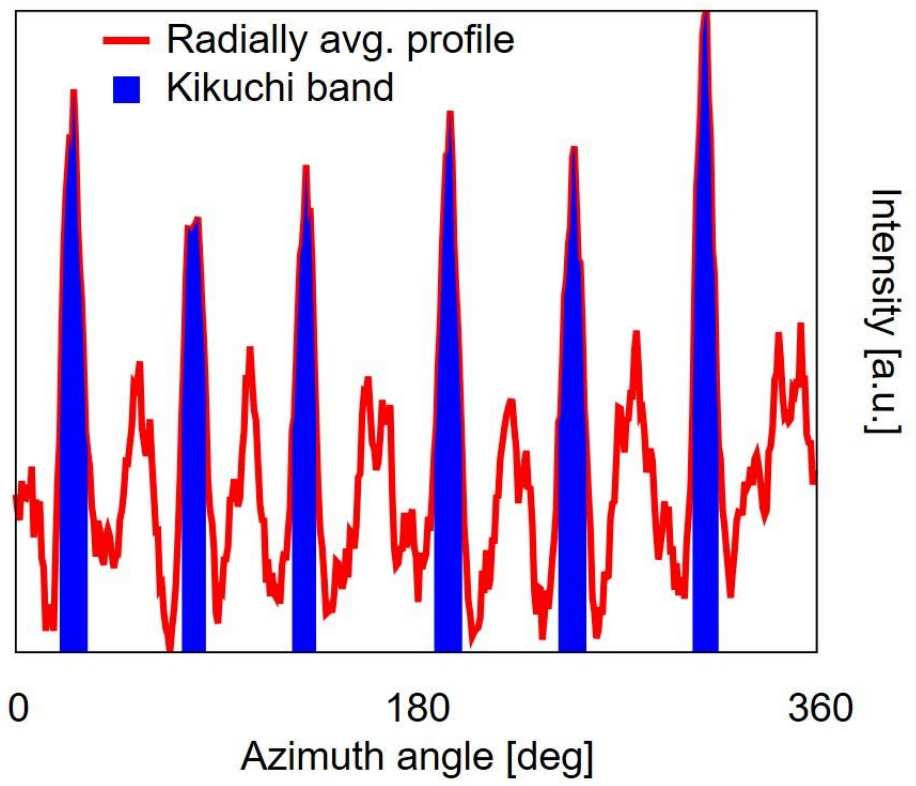

Figure 1. Measuring orientation by Kikuchi bands. (a) CBED pattern with renormalized high-angle annular area dominated by Kikuchi band signal. The green cross marks the central beam, the red dot marks the band intersection. (b) Azimuthal scan in the selected dark-field area. The six peaks correspond to the three brightest Kikuchi bands. From their positions, the associated band crossing is deduced by geometry.
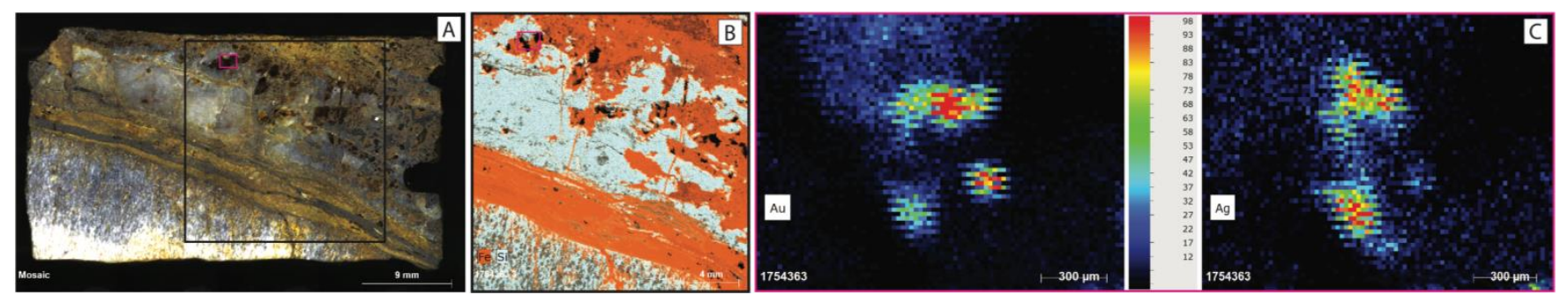

Figure 2. Orientation mapping over a twin boundary in steel. (a) Synthetic high-resolution HAADF image showing a horizontal twin boundary. An in-plane rotation of $\alpha=70.6^{\circ} \pm 0.2^{\circ}$ was measured. (b) Map of local out-of-plane crystal tilt indicating sample bending of a few milliradians over the field of view.

\section{References}

[1] T. Grieb et al., Ultramicroscopy 190 (2018)

[2] K.E. MacArthur et al., Microsc. \& Microanal. Accepted

[3] Funding: Jülich Melbourne Postgraduate Academy (JUMPA, M.C.), Helmholtz Contract VH-NG1317 (K. M-C.), DFG HE 7192/1-2 (K. M.). EMPAD data has been recorded within a cooperation with Thermo Fisher Scientific, Inc. 